\title{
Anterior Circulation Acute Ischemic Stroke Associated with Atherosclerotic Lesions of the Cervical ICA: A Nosologic Entity Apart
}

(D).F. Eker, (DP. Panni, (D). Dargazanli, (D) Marnat, (D). Arquizan, (DP. Machi, DI. Mourand, DG. Gascou, (DE. Le Bars,

(D) V. Costalat, and (D) A. Bonafé

\begin{abstract}
BACKGROUND AND PURPOSE: Mechanical thrombectomy for patients with acute ischemic stroke with tandem occlusions has been shown to present varying reperfusion successes and clinical outcomes. However, the heterogeneity of tandem occlusion etiology has been strongly neglected in previous studies. We retrospectively investigated patients with acute ischemic stroke atherothrombotic tandem occlusion.

MATERIALS AND METHODS: All consecutive patients with acute ischemic stroke with atherothrombotic tandem occlusions treated with mechanical thrombectomy in our center between September 2009 and April 2015 were analyzed. They were compared with patients with acute ischemic stroke with dissection-related tandem occlusion and isolated intracranial occlusion treated during the same period. Comparative univariate and multivariate analyses were conducted, including demographic data, safety, and rates of successful recanalization and good clinical outcome.

RESULTS: Despite comparable baseline severity of neurologic deficits and infarct core extension, patients with atherothrombotic tandem occlusions were older $(P<.001)$, were more frequently smokers $(P<.001)$, and had globally more cardiovascular risk factors $(P<.001)$ than the other 2 groups of patients. The patients with atherothrombotic tandem occlusions had significantly longer procedural times $(P<.001)$, lower recanalization rates $(P=.004)$, and higher global burden of procedural complications $(P<.001)$. In this group, procedural complications $(\mathrm{OR}=0.15, P=.02)$ and the $\mathrm{TICl} 2 \mathrm{~b} / 3$ reperfusion scores $(\mathrm{OR}=17.76, P=.002)$ were independently predictive factors of favorable clinical outcome.
\end{abstract}

CONCLUSIONS: Our study suggests that atherothrombotic tandem occlusions represent a peculiar and different nosologic entity compared with dissection-related tandem occlusions. This challenging cause of acute ischemic stroke should be differentiated from other etiologies in patient management in future prospective studies.

ABBREVIATIONS: AIS = acute ischemic stroke; $\mathrm{CSA}=$ carotid stent placement and angioplasty; $\mathrm{MT}=$ mechanical thrombectomy; sICH = symptomatic intracranial hemorrhage; $\mathrm{TO}=$ tandem occlusion

$\mathbf{T}$ he benefits of mechanical thrombectomy (MT) in reperfusion success, safety, and clinical outcome in patients with acute ischemic stroke (AIS) have been demonstrated by several randomized trials. ${ }^{1-5}$ These trials concerned mostly patients with intracranial large-vessel occlusion in the anterior circulation. However, up to

Received February 16, 2017; accepted after revision June 9.

From the Service de Neuroradiologie (O.F.E., P.P., C.D., P.M., G.G., E.L.B., V.C., A.B.) and Service de Neurologie (C.A.), Hôpital Gui de Chauliac, CHRU de Montpellier, Montpellier, France; Department of Neurosurgery and Radiosurgery (P.P.), San Raffaele University Hospital, Milan, Italy; and Service de Neuroradiologie (G.M., I.M.), Hôpital Pellegrin, CHRU de Bordeaux, Bordeaux, France.

Please address correspondence to O.F. Eker, MD, PhD, Department of Interventional Neuroradiology, Gui de Chauliac Hospital, CHRU de Montpellier, 80 Ave Augustin Fliche, 34295 Montpellier cedex 9, France; e-mail: eker_omer@yahoo.fr

$20 \%$ of patients with an intracranial large-vessel occlusion also present with an additional occlusion of the extracranial ICA, mainly due to atherosclerosis or dissection and less frequently to cardioembolism or undetermined etiology. ${ }^{6,7}$ These so-called tandem occlusions (TOs) have poorer prognosis in reperfusion and clinical outcome after IV fibrinolysis, ${ }^{7-10}$ probably because of poor response to fibrinolysis and/or the high clot burden associated with them. They are considered the worst-case scenarios among patients with anterior circulation stroke, leading to severe neurologic morbidity and death in up to $70 \%$ and $55 \%$ of cases, respectively. ${ }^{11-13}$

The available data on TOs treated with MT show varying rates of reperfusion success (ranging from $62.5 \%$ to $100 \%$ ) and favorable outcome at 3 months (ranging from $29.2 \%$ to $54 \%$ of patients with mRS 0-2). ${ }^{7,13-15}$ Many factors may explain these varying results, such as the following: 1) the low incidence of TOs and their systematic exclusion or limited inclusion in most of the main clinical trials of 


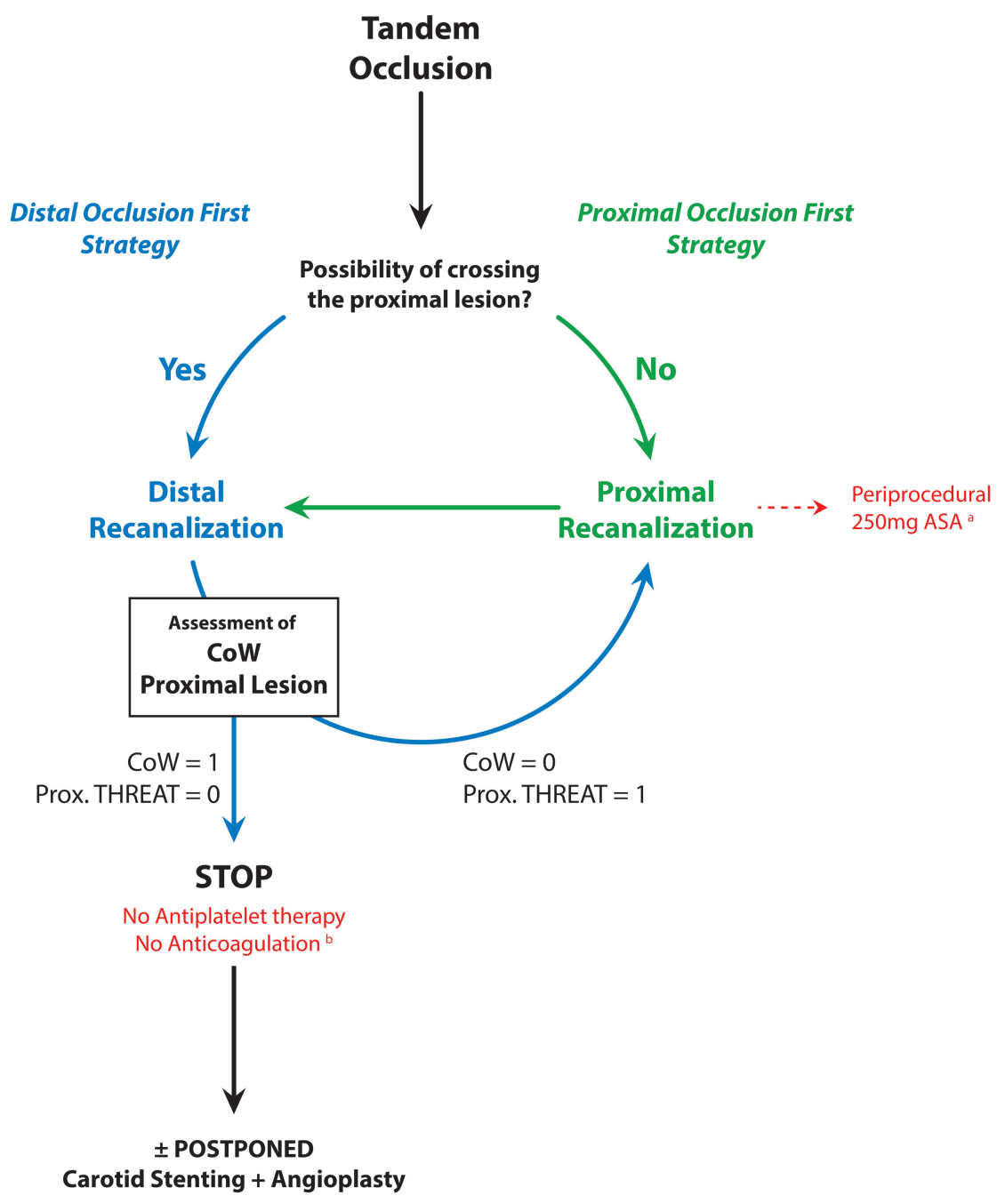

FIG 1. Algorithm of endovascular therapeutic strategies for tandem occlusions. The absence $(n=$ $0)$ or presence $(n=1)$ condition the proximal treatment or not. The algorithm of the therapeutic decisions describes 2 strategies consisting of the treatment of, first, the proximal lesion (proximal occlusion strategy [POF]) or, first, the distal occlusion (distal occlusion first strategy [DOF]). The decision between the POF or DOF strategies is based on the following considerations raised during the endovascular procedure: 1) the ease of crossing the proximal occlusion site (easy crossing? difficult or impossible crossing?); 2) the patency and efficiency of the circle of Willis (efficient CoW or absence of CoW?); and 3) how threatening is the proximal atherosclerotic lesion? (unstable ulcerated plaque? moderate regular plaque?). In thrombus on ulcerated plaque, usually a single thromboaspiration allows easily crossing the proximal occlusion site and treating the distal (ie, intracranial) occlusion. In case of POF (a), the antiplatelet regimen consisted of the periprocedural intravenous administration of a unique loading dose of aspirin $(250 \mathrm{mg})$ until the first imaging follow-up at 24 hours postoperatively. After ruling out any hemorrhagic transformation at 24 hours, a daily dual-antiplatelet therapy ( $160 \mathrm{mg}$ of aspirin $+75 \mathrm{mg}$ of clopidogrel) was instituted. In case of DOF (b), no anticoagulation or antiplatelet therapies were administered until the first imaging follow-up at 24 hours postoperatively. After we ruled out any hemorrhagic transformation at 24 hours, a daily monoantiplatelet therapy (160 mg of aspirin) was instituted. CoW indicates circle of Willis (efficient $=1$; nonefficient or absent $=0$ ); Prox. THREAT indicates any threatening of the proximal lesion (either atherosclerotic or dissecting).

MT for AIS; 2) the limitation of the previously reported data to retrospective small case series with heterogeneous treatment strategies; and 3 ) the heterogeneity of TO etiologies that has been strongly neglected and may have considerably biased the results. A recent metaanalysis of individualized patient data from the 5 main randomized trials confirmed the benefit of MT for patients with TOs, though the heterogeneity of treatment methods challenges substantially the chances of defining a standardized optimal treatment method. ${ }^{16}$

In this work, we evaluated the efficacy and safety of MT for the treatment of patients with AIS caused by atherothrombotic TO. The results of MT in atherothrombotic TO were secondarily compared to those obtained for patients with dissection-related $\mathrm{TO}$ with isolated intracranial occlusion.

\section{MATERIALS AND METHODS Patient Selection}

Data on patients treated with MT for AIS due to atherothrombotic TO between September 2009 and April 2015 were retrospectively extracted from a prospectively maintained data base. The decision for treatment was based on the clinical/radiologic severity mismatch defined as the discrepancy between the NIHSS score and the baseline core infarct size estimated on the DWIASPECTS. Age and time after symptom onset were not absolute exclusion criteria. The patient's eligibility for MT was based on the following criteria: $\mathrm{mRS}$ 0-2 before the AIS, NIHSS score of $\geq 8$ or fluctuating symptoms, intracranial large-vessel occlusion, and DWIASPECTS of $\geq 4$. When it was not contraindicated, patients received IV rtPA at the usual dose of $0.9 \mathrm{mg} / \mathrm{kg}$ before or concomitantly with MT. The study was approved by the local institutional review board. All patients underwent initial MR imaging with a $1.5 \mathrm{~T}$ or $3 \mathrm{~T}$ whole-body scanner (Avanto or Skyra system; Siemens, Erlangen, Germany). The imaging protocol included DWI, FLAIR, gradient-echo T2* imaging, and contrast-enhanced MRA.

\section{Endovascular Procedure and Postintervention Medications}

The technical aspects of the endovascular procedure are detailed in the On-line Appendix. A "distal occlusion first treatment strategy" was performed if the proximal cervical lesion could be crossed without problems. After achieving the intracranial reperfusion, we assessed the circle of Willis functionality by opacification of the other supra-aortic trunks. In the case of ineffective circle of Willis supply to the recanalized MCA, a stent was placed across the proximal occlusion. A stent was inserted on a case-by-case basis. In the case of highly stenotic lesions that could not initially be broken through, a "proximal occlusion first" treatment strategy was performed. The proximal lesion was primarily treated by carotid angioplasty and stent placement. Then, intracranial thrombectomy was performed. Figure 1 illustrates the management algorithm for tandem occlusions in our center. All treatments were performed with the patient under general 
anesthesia in an operating room with a biplane angiography system (Allura; Philips Healthcare, Best, Netherlands). No heparin was administered before, during, or after the MT. If no hemorrhagic transformation of the infarct was observed at 24 hours, treatment with 160 mg of aspirin daily was initiated. In the case of carotid stent placement and angioplasty (CSA), before the stent placement, patients were given a single IV load of aspirin $(250 \mathrm{mg})$ until the first imaging follow-up (CT or MR imaging) at 24 hours. In the absence of hemorrhagic transformation, a daily dose of $75 \mathrm{mg}$ of aspirin was then maintained in association with $75 \mathrm{mg}$ of clopidogrel for 3 months. Moreover, blood pressure was strictly monitored and pharmacologically controlled to maintain a systolic blood pressure of $<140 \mathrm{~mm}$ $\mathrm{Hg}$ after revascularization because of the risk of hyperperfusion of the ipsilateral hemisphere.

\section{Analyzed Variables}

Clinical variables included demographic data, history of hypertension, dyslipidemia, diabetes, cigarette smoking, baseline NIHSS score, and clinical outcome at 3 months assessed by a stroke neurologist not involved in the initial patient management. Imaging data included the ischemic stroke side, DWI-ASPECTS, intracranial occlusion site (intracranial ICA, MCA, M1 or M2 segment, and/or anterior cerebral artery), occurrence of hemorrhagic transformation, and infarct core extension on the 24-hour postprocedure CT or MR imaging scan. Hemorrhagic transformation was categorized according to the European Cooperative Acute Stroke Study criteria (H-1, H-2, PH-1, and PH-2). ${ }^{17}$ Symptomatic intracranial hemorrhage $(\mathrm{sICH})$ was defined as a CT- or MR imaging-documented hemorrhage that occurred within 36 hours after treatment onset and was temporally related to a NIHSS worsening of 4 points. Procedural data included the time between symptom onset and reperfusion (onset-to-reperfusion time); the procedural time (ie, from groin puncture to reperfusion); the administration of IV rtPA; the use of stent retrievers for intracranial occlusions and of angioplasty and/or stent placement for extracranial occlusions; recanalization strategy; the final reperfusion outcome (successful reperfusion defined as TICI $2 \mathrm{~b} / 3$ score), ${ }^{18}$ and procedural complications. These included distal embolus defined as an occlusive embolus in a previously uninvolved territory, vessel dissection, intraparenchymal hemorrhage, device detachment, and severe vasospasm that needed intraarterial nimodipine administration. Because the study population was limited, only a comparative analysis of the treatment strategies was conducted (ie, proximal occlusion first strategy versus distal occlusion first strategy) rather than a comparison of the different endovascular techniques (eg, percutaneous transarterial angioplasty only versus stent percutaneous transarterial angioplasty).

In addition, we recorded the following data: prior antiplatelet or anticoagulant therapies, stenosis degree on DSA images (graded as $0=$ occlusive plaque, $1=$ severe stenotic plaque [ $>70 \%$ of stenosis], $2=$ moderate stenotic plaque with occlusive thrombus $[<70 \%$ of stenosis], and $3=$ nonstenotic but ulcerated plaque with occlusive thrombus), and the occurrence of extracranial intrastent thrombosis during the first week after the treatment.

\section{Statistical Analysis}

Continuous values were expressed as medians and interquartile ranges or as means $\pm \mathrm{SD}$, according to their distribution. Categoric variables were expressed as counts and percentages. Differences between categoric variables were analyzed with the Fisher exact or the Kruskal-Wallis rank sum test. Differences among continuous variables were analyzed with the Student $t$ or the Mann-Whitney $U$ test according to the results of the normality test. To identify factors contributing to the clinical outcome, we analyzed several covariables in the subgroups of patients with mRS $0-2$ versus mRS $>2$. A $P$ value $<.05$ was considered as statistically significant. Variables with $P$ values $<.10$ from the univariate analysis were considered candidate predictors of a favorable/unfavorable outcome and were entered in the multivariate analysis that used a logistic regression model. Patients with atherothrombotic TO were compared with patients with dissection-related TO $(n=20)$ and isolated intracranial occlusion $(n=$ 201) from the same data base and the same period. Data on the last 2 groups were previously published. ${ }^{19}$ The statistical analyses were performed with R Statistical and Computing Software, Version 3.2.1 (http://www.r-project.org/). ${ }^{20}$

\section{RESULTS}

Five hundred eighteen consecutive patients with acute intracranial artery occlusion in the anterior circulation were admitted to our institution and treated with MT during the study period. Among these patients, $66(12.7 \%)$ had an additional atherothrombotic lesion of the extracranial ICA. The demographic, procedural, and outcome data in this group as well as the data of dissection-related TO and isolated intracranial occlusion groups are reported in On-line Table 1. In the atherothrombotic TO group, DSA-based evaluation of the atheromatous carotid lesions showed the presence of grade 0 plaque in $39(59.1 \%)$, grade 1 plaque in $17(25.8 \%)$, grade 2 plaque in $4(6.1 \%)$, and grade 3 plaque in $6(9.1 \%)$ patients. The distributions of the stenosis degree among the subgroups "emergent CSA, proximal occlusion first strategy," "emergent CSA, distal occlusion first strategy," "postponed CSA," and "no CSA" were not statistically different $(P=.09$; the results are reported in On-line Table 2).

\section{Recanalization Strategies}

MT was performed in 65 patients $(98.5 \%)$ with stent retrievers. Aspiration thrombectomy alone was performed in only 1 patient (1.5\%), and proximal or distal aspiration thrombectomy was performed concomitantly with stent retrieval in 48 patients (72.7\%). Two patients (3\%) underwent intracranial stent placement. Extracranial acute balloon angioplasty alone was performed in 19 patients (28.9\%). Emergent CSA was achieved in 26 (39.4\%) patients among whom proximal occlusion first and distal occlusion first strategies were used in $10(38.5 \%)$ and $16(61.5 \%)$ patients, respectively. In the proximal occlusion first group, the cervical plaques were graded as 1 in $7(70 \%)$ and 2 in $3(30 \%)$ patients. In the distal occlusion first group, the cervical plaques were graded as 1 in $9(56.3 \%), 2$ in $3(18.7 \%), 3$ in 2 (12.5\%), and 4 in $2(12.5 \%)$ patients. Planned (or postponed) CSA was required in 14 patients (21.2\%) 1 week later, with stenosis degree graded as 0 in 9 (64.3\%) and 1 in $5(35.7 \%)$ patients. 
Table 1: Univariate analysis in the atherothrombotic tandem occlusion group

\begin{tabular}{lccc}
\hline & $\begin{array}{c}\text { mRS 0-2 }(\boldsymbol{n}=\mathbf{2 7}) \\
\text { No. (\%) or } \\
\text { (Mean) }\end{array}$ & $\begin{array}{c}\text { mRS }>\mathbf{2}(\boldsymbol{n}=\mathbf{3 8}) \\
\text { No. (\%) or } \\
\text { (Mean) }\end{array}$ & $\begin{array}{c}\boldsymbol{P} \\
\text { Value }\end{array}$ \\
\hline Age (yr) & $27(64.6 \pm 8.1)$ & $38(69.2 \pm 10.6)$ & .04 \\
Men & $27(0.9 \pm 0.3)$ & $38(0.9 \pm 0.3)$ & .47 \\
Hypertension & $21(77.8)$ & $27(71.1)$ & .58 \\
Cigarette smoking & $11(40.7)$ & $21(55.3)$ & .32 \\
Dyslipidemia & $19(70.4)$ & $19(50)$ & .13 \\
Diabetes & $11(40.7)$ & $14(36.8)$ & .80 \\
Other cardiovascular risk factors & $2(7.4)$ & $9(23.7)$ & .11 \\
Left side & $13(48.1)$ & $27(71.1)$ & .08 \\
Distal ICA occlusion & $8(29.6)$ & $22(57.9)$ & .04 \\
Baseline NIHSS score & $27(14.9 \pm 6.3)$ & $38(18.9 \pm 5.2)$ & .005 \\
Baseline DWI-ASPECTS & $27(7.1 \pm 1.7)$ & $37(6.7 \pm 1.5)$ & .22 \\
IV rtPA & $16(59.3)$ & $24(63.2)$ & .80 \\
Onset-reperfusion time (min) & $24(377 \pm 147.3)$ & $32(390.4 \pm 173.4)$ & .93 \\
Procedural time (min) & $25(76.6 \pm 39.1)$ & $33(85.8 \pm 48.5)$ & .49 \\
Number of stent retriever passes & $27(1.9 \pm 0.9)$ & $37(2.5 \pm 1.2)$ & .03 \\
Acute stenting & $10(37)$ & $15(39.5)$ & 1 \\
Procedural complications & $5(18.5)$ & $17(44.7)$ & .04 \\
Ischemic extension & $2(7.4)$ & $19(50)$ & $<.001$ \\
Hemorrhagic transformation & $8(29.6)$ & $23(60.5)$ & .02 \\
Subarachnoid hemorrhage & $1(3.7)$ & $4(10.5)$ & .39 \\
H-1 & $3(11.1)$ & $3(7.9)$ & .69 \\
H-2 & $4(14.8)$ & $7(18.4)$ & .75 \\
PH-1 & $0(0)$ & $3(7.9)$ & .26 \\
PH-2 & $0(0)$ & $7(18.4)$ & .04 \\
sICH & $0(0)$ & $10(26.3)$ & .004 \\
TICl 2b/3 & $25(92.6)$ & $16(42.1)$ & \\
\hline & & & \\
\hline
\end{tabular}

patients available for the outcome analysis (Table 1). At the 3-month followup, 27 patients $(41.5 \%)$ were mRS $0-2$ and $15(23.1 \%)$ were mRS 6, among whom 13 (20\%) died before discharge. Favorable outcome at 3 months was significantly associated with younger age $(P=.04)$, lower NIHSS score $(P=$ $.005)$, and TICI $2 \mathrm{~b} / 3$ reperfusion $(P<$ $.001)$. Poor clinical outcome was associated with intracranial ICA occlusion $(P=.04)$, TICI $0-2$ a reperfusion $(P<$ $.001)$, occurrence of procedural complications $(P=.03)$, infarct core extension $(P<.001)$, and $\operatorname{siCH}(P=.004)$ at day 1 . The baseline NIHSS score was moderately but significantly correlated with the mRS score at 3 months $(R=0.36$, $P=.003)$, whereas the DWI-ASPECTS was not $(P=.21)$. The onset-reperfusion and procedural times did not influence the clinical outcome $(P=.93$ and $P=.49$, respectively). The recanalization strategy did not influence the rates of successful reperfusion $(P=1)$ or of favorable clinical outcome at 3 months $(P=.66)$. However, proximal occlusion

\section{Reperfusion and Safety}

TICI $2 b / 3$ reperfusion was significantly associated with shorter procedural times $(P=.03)$ and higher rates of distal MCA occlusions $(P=.01)$. The onset-reperfusion times were longer in patients with TICI $0-2 \mathrm{a}$ scores than in those with TICI $2 \mathrm{~b} / 3$ scores (449 \pm 189 minutes versus $356 \pm 137$ minutes), but this difference was not significant $(P=.06)$. Reperfusion failure was significantly associated with higher infarct core extension $(P=.03)$ and $\operatorname{sICH}(P=.03)$ occurrence. Procedural complications occurred in 22 patients $(33.3 \%)$. They included the following: acute intrastent thrombosis $(n=1,1.5 \%)$, distal embolus $(n=6,9.1 \%)$, vessel dissection $(n=4,6.1 \%)$, intraparenchymal hemorrhage due to vessel perforation $(n=2,3 \%)$, stent-retriever rupture $(n=1$, $1.5 \%)$, and severe vasospasm of the cervical or intracranial arteries that required intra-arterial nimodipine infusion $(n=8$, $12.1 \%)$. Day 1 control imaging showed hemorrhagic transformation in 28 patients $(42.4 \%)$, of whom 10 (15.2\%) were symptomatic and were classified as $\mathrm{PH}-1$ or $\mathrm{PH}-2$. sICH was significantly associated with a history of hypertension $(P=.01)$, diabetes $(P=$ $.05)$, and reperfusion failure $(P=.03)$. In the patients who underwent postponed CSA $(n=14), 4$ patients had an intrastent thrombosis at follow-up imaging (within 1 week or delayed thrombosis), resulting in 5 intrastent thromboses in our series (20\% in acute setting and $80 \%$ in a delayed manner). In all 5 patients, TICI $2 \mathrm{~b} / 3$ reperfusion was achieved. In only 2 patients (40\%), intrastent thrombosis was associated with clinical worsening at 3-month follow-up (mRS 4 and 6, respectively).

\section{Clinical Outcome}

At 7 days, infarct core extension was observed in 21 patients (31.8\%). One patient was lost to 3-month follow-up, leaving 65 first was significantly associated with a higher $\operatorname{siCH}$ rate $(P=.01)$ at day 1 but did not influence the infarct core extension $(P=.33)$. Previous antiplatelet or anticoagulation therapies $(n=15)$ and IV rtPA administration $(n=40)$ did not affect the rates of successful reperfusion, sICH, infarct core extension, or favorable clinical outcome at 3 months. However, previous antiplatelet or anticoagulation therapies $(n=15)$ were associated with higher rates of PH-2 hemorrhages at day $1(n=4,26.6 \%$ versus $n=3 / 51,5.9 \%$ in patients without these treatments; $P=.04)$ (On-line Table 3).

\section{Comparison of the Atherothrombotic TO Group with the Dissection-Related TO and Isolated Intracranial Occlusion Groups}

Compared with the dissection-related TO group, patients in the atherothrombotic TO group were significantly older $(P<.001)$ and more frequently cigarette smokers $(P=.01)$. They had significantly higher proportions of intracranial ICA termination occlusion $(P=.001)$ and procedural complications $(P=.05)$ and a significantly lower proportion of $\mathrm{mRS} 0-2$ at 3 months $(P=.04)$ (On-line Table 4). Compared with the isolated intracranial occlusion group, they were more frequently men $(P<.001)$ and cigarette smokers $(P<.001)$. They had significantly longer onsetreperfusion times $(P=.05)$ and procedural times $(P<.001)$, lower TICI $2 \mathrm{~b} / 3$ reperfusion $(P=.002)$, higher complication rates $(P<.001)$, and higher sICH rates $(P=.01)$, and especially $\mathrm{PH}-2$ hemorrhages $(P=.02)$. Patients with atherothrombotic TO also had a markedly lower proportion of those with mRS $0-2$ and a higher proportion of those with mRS 6, despite the lack of statistical significance (On-line Table 5). 
Table 2: Multivariate analysis of good clinical outcome predictors

\begin{tabular}{lcccc}
\multicolumn{1}{c}{ Groups, Variables } & $\begin{array}{c}\text { Odds } \\
\text { Ratio }\end{array}$ & $\begin{array}{c}95 \% \\
\text { Lower Cl }\end{array}$ & $\begin{array}{c}95 \% \\
\text { Upper Cl }\end{array}$ & $\begin{array}{c}\boldsymbol{P} \\
\text { Value }\end{array}$ \\
\hline ATO & & & & \\
Age & 0.93 & 0.85 & 1.00 & .07 \\
Baseline NIHSS score & 0.89 & 0.79 & 1.00 & .06 \\
Intracranial ICA occlusion & 0.39 & 0.09 & 1.63 & .20 \\
Periprocedural complications & 0.15 & 0.03 & 0.74 & .02 \\
TICl 2b/3 & 17.76 & 2.90 & 108.81 & .002 \\
IICO & & & & \\
Age & 0.95 & 0.93 & 0.98 & $<.001$ \\
Baseline NIHSS score & 0.91 & 0.85 & 0.97 & .004 \\
Intracranial ICA occlusion & 1.33 & 0.63 & 2.83 & .45 \\
Periprocedural complications & 1.36 & 0.50 & 3.70 & .55 \\
TICI 2b/3 & 3.57 & 1.48 & 8.61 & .005 \\
\hline
\end{tabular}

Note:-ATO indicates atherothrombotic tandem occlusion; IICO, isolated intracranial occlusion. a Previously published. ${ }^{19}$

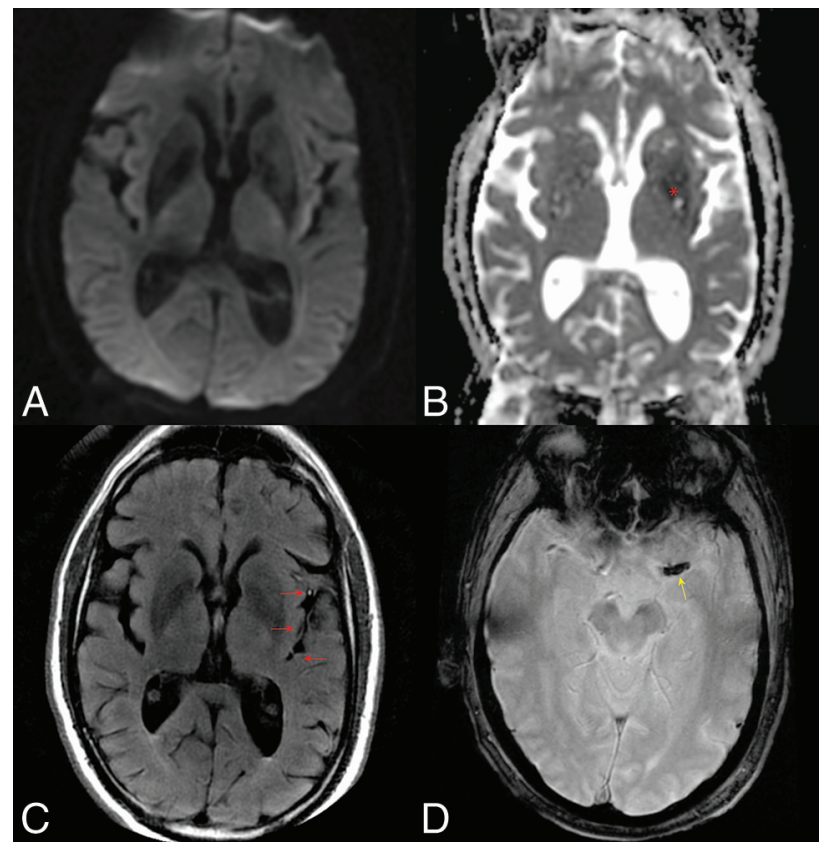

FIG 2. Baseline MR imaging. A 69-year-old patient was admitted for a left middle cerebral artery ischemic stroke, with an NIHSS score of 18 at 3 hours after symptom onset. The DWI and ADC maps ( $A$ and $B$, red asterisk) showed a limited infarct core of the lenticular nucleus, negative findings on FLAIR, and a clot in the Ml segment of the artery with susceptibility artifacts on the T2 echo gradient ( $D$, yellow arrow) acquisition. Some evidence of slow flow was also visible on FLAIR as sulcal hypersignals ( $C$, red arrows).

Due to the small number of patients in the dissection-related TO group, a multivariate logistic regression analysis was performed for only the atherothrombotic TO and isolated intracranial occlusion groups (Table 2). In the atherothrombotic TO group, only procedural complications $(P=.02)$ and TICI $2 \mathrm{~b} / 3$ reperfusion $(P=.002)$ were independent predictors of favorable clinical outcome at 3 months. Whereas in the isolated intracranial occlusion group, only younger age $(P<.001)$, baseline NIHSS score $(P=.004)$, and TICI $2 \mathrm{~b} / 3$ reperfusion $(P=.005)$ were independent predictors of favorable clinical outcome at 3 months.

Figs 2-4 illustrate a case of AIS due to an atherothrombotic TO. In On-line Fig 1, the main results are reported as a flow chart.

\section{DISCUSSION}

The available data on TOs treated with MT are limited due to their low incidence, the heterogeneity of TO etiologies, and treatment strategies in the reported case series. In this study, we analyzed a relatively large series of patients with solely atherothrombotic TO treated with MT in terms of reperfusion rate, safety, and clinical outcome as well as the predictors of favorable outcome in this group. Then we compared this group with patients with dissection-related TOs or with isolated intracranial occlusions from the same AIS data base during the same period.

Two important elements emerge from our study. Our results are in good agreement with previously reported findings. ${ }^{7,13-16}$ Despite its retrospective feature, our rigorous methodologic approach strongly supports the previously suggested idea that atherothrombotic TOs represent a peculiar and different nosologic entity of AIS compared with dissection-related TOs and isolated intracranial occlusions. Indeed, despite comparable baseline NIHSS and DWI-ASPECTS distributions, patients with atherothrombotic TO were significantly older and more frequently smokers than patients with dissection-related TOs. They presented globally with more cardiovascular risk factors. Second, atherothrombotic TOs represent an important technical challenge that affects more dramatically the clinical outcome than isolated intracranial occlusions and dissection-related TOs. This is highlighted by the significantly longer procedural times, lower rates of successful reperfusion, and lower rates of favorable clinical outcomes in this group. This might be partly explained by the significantly higher rate of intracranial ICA occlusion and the higher global burden of procedural complications in the atherothrombotic TO group. The first is often associated with bigger clots (ie, technically difficult to retrieve) and an increased probability of collateral circulation impairment due to decreased flow in the anterior cerebral artery. The multivariate analysis supported these results by showing that in patients with atherothrombotic TO, procedural complications $(\mathrm{OR}=0.15, P=.02)$ and TICI $2 \mathrm{~b} / 3$ reperfusion $(\mathrm{OR}=17.76, P=.002)$ were the 2 key elements independently predictive of favorable outcome at the 3-month follow-up.

In our study, the distinction of TOs according to their etiologies revealed a higher rate of complications (33\%) compared to that previously reported in large series (from $0 \%$ to $21.6 \%$ ). Whereas in dissection-related TOs group, the complication rate was markedly lower (Table 3).,13-15,21-27 This finding might be due to the design of previous analyses and because we also included severe vasospasms needing the intra-arterial administration of nimodipine. However, in this work, the different patient subgroups were homogeneous, whereas the previously reported studies mostly combined different types of TOs with various ratios of atherothrombotic-/dissection-related TOs. This latter methodologic approach could be confusing and a source of bias in the analysis of populations as well as in the evaluation and devel- 


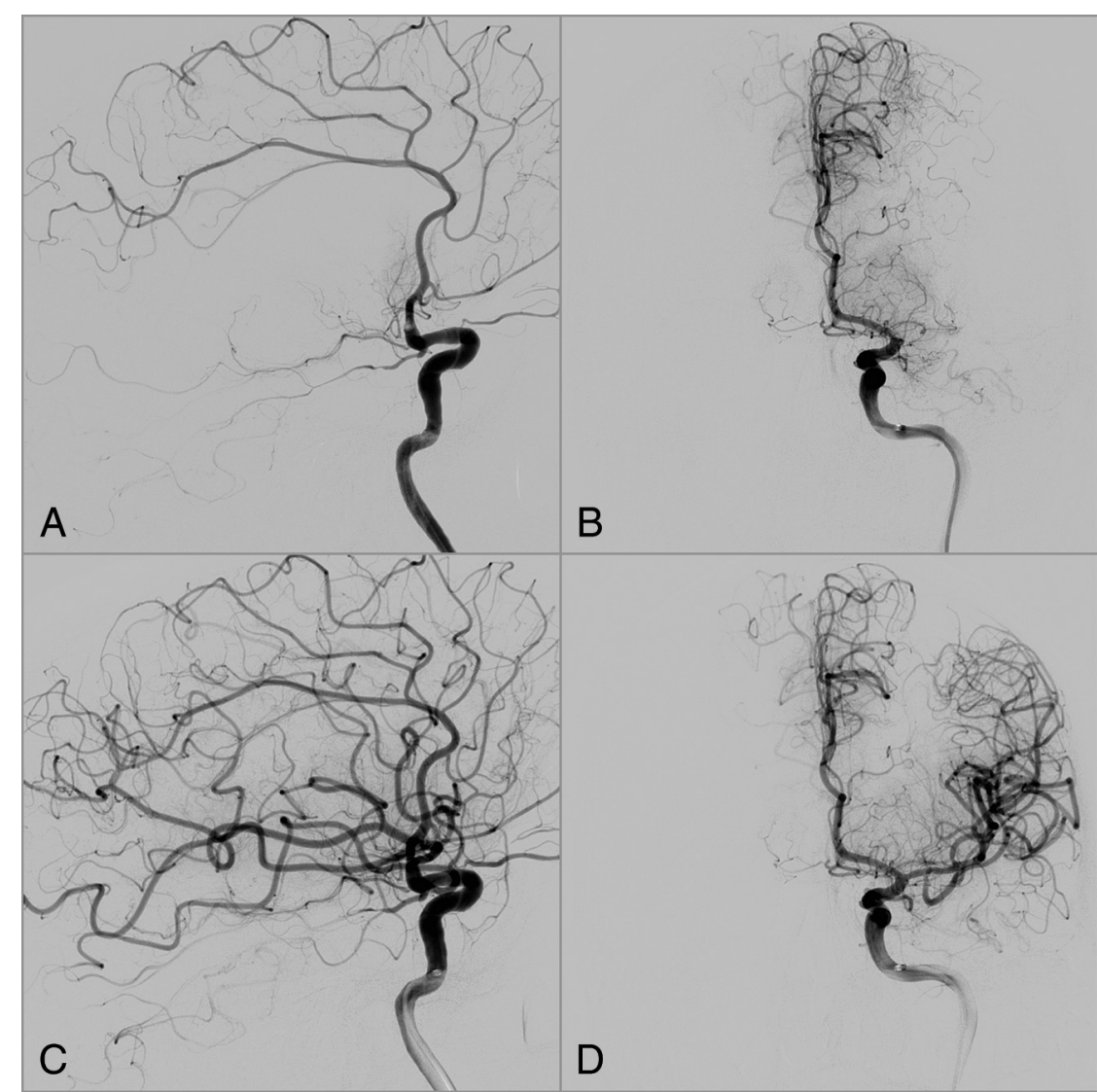

FIG 3. DSA of intracranial reperfusion. The same patient as in Fig 2 underwent mechanical thrombectomy following a distal occlusion first recanalization strategy. The figure shows the angiogram of the intracranial vasculature of left internal carotid artery before and after MT with a stent retriever. Before MT $(A$ and $B)$, the angiogram shows the occlusion of left middle cerebral artery (segment Mi) with a $\mathrm{TICl}$ score of 0 and a poor pial collaterality from the anterior cerebral artery. MT allowed complete reperfusion of the MCA territory with a $\mathrm{TICl}$ score of 3 ( $C$ and $D$ ) without any distal emboli.

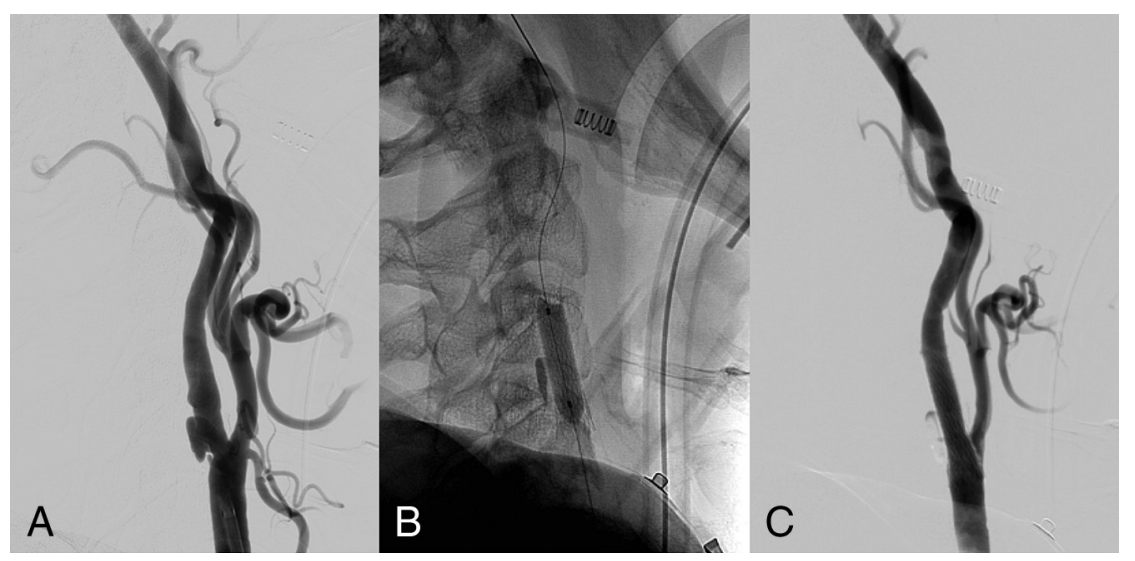

FIG 4. DSA of proximal carotid treatment. After full reperfusion of the intracranial occluded vessel, the patient underwent a stent placement and angioplasty of the extracranial carotid artery. The DSA shows an ulcerated atherosclerotic plaque of the carotid bulb before the treatment $(A)$. After the angioplasty $(B)$, a full recanalization of the vessel was achieved $(C)$.

opment of new management strategies. Indeed, it leads to an inappropriate averaging of the results.

There is no consensus concerning the recanalization sequence strategy for TOs. Both proximal occlusion first and distal occlusion first approaches have their variations, advantages, and shortcomings. ${ }^{7,21,26}$ In our series, the technique was chosen by the operator on the basis of the proximal lesion and varied with the improvement and availability of new endovascular techniques. Therefore and given the limited number of patients, our work was not able to address this point. However, the proximal occlusion first approach was significantly associated with markedly higher rates of sICH and, less significantly, with infarct core extension at day 1 compared with the distal occlusion first approach. Conversely, both approaches did not differ in the rates of reperfusion and favorable outcome at 3 months.

Atherothrombotic tandem occlusions represent an important technical challenge that affects, more dramatically, the clinical outcome than dissection-related tandem occlusions and isolated intracranial occlusions. This is clearly highlighted by the significantly longer procedural times, lower recanalization rates, and higher global burden of procedural complications in this group. Therefore, alternative therapeutic approaches may be discussed. Few authors proposed emergent endarterectomy for patients with AIS. ${ }^{28}$ However, their results remain confidential and concerned limited numbers of patients $(n=6)$ with a high risk of fatal hemorrhagic transformation. Similarly, the use of abciximab for intrastent thrombosis has been suggested by other authors. ${ }^{29}$ We did not use this drug in our patients with intrastent thrombosis because of the high risk of hemorrhagic transformation previously reported. ${ }^{30}$

Most interesting, in the atherothrombotic TO group, IV rtPA administration did not affect the rate of successful reperfusion and of hemorrhagic transformation or the outcome at 3 months. Although this work does not allow us to draw any conclusions, given the previously mentioned limitations, this observation questions the benefit of IV rtPA in this specific group of patients. Conversely, antiplatelet and/or anticoagulant drugs before AIS significantly increased the occurrence of $\mathrm{PH}-2$ hemorrhages at day $1(P=.04)$. These treatments should be considered with caution, especially in the postoperative management of these patients, to prevent the risk of intracranial bleeding.

Our findings are in good agreement with the HERMES collaboration group meta-analysis ${ }^{16}$ in term of good clinical outcome at 
Table 3: Summary of previously published tandem occlusion series

\begin{tabular}{|c|c|c|c|c|c|c|c|}
\hline $\begin{array}{l}\text { Authors, Year } \\
\text { Published }\end{array}$ & $\begin{array}{l}\text { Patients } \\
\text { (No.) }\end{array}$ & $\begin{array}{c}\% \text { of TO } \\
\text { Etiologies }^{\mathrm{a}}\end{array}$ & $\begin{array}{l}\text { TICI } 2 b / 3 \text { or } \\
\text { TIMI } 2-3\end{array}$ & $\mathrm{mRS} \leq 2$ & $\mathrm{mRS} 6$ & sICH & $\begin{array}{l}\text { Periprocedura } \\
\text { Complications }\end{array}$ \\
\hline Malik et al, $2011^{21}$ & 77 & $100 / 0 / 0 / 0$ & $58.0(75.3)^{\mathrm{b}}$ & $32.0(41.6)$ & $18.0(23.4)$ & $8.0(10.4)$ & NA \\
\hline Kwak et al, $2013^{2}$ & 35 & $100 / 0 / 0 / 0$ & $26.0(74.3)$ & $22.0(62.9)$ & 4.0 (11.4) & $1.0(2.9)$ & $0.0(0.0)$ \\
\hline Stampfl et al, $2014^{23}$ & 24 & $100 / 0 / 0 / 0$ & $15.0(62.5)$ & $7.0(29.2)$ & 4.0 (16.7) & 4.0 (16.7) & $1.0(4.2)$ \\
\hline Maurer et al, $2015^{24}$ & 43 & $77 / 7 / 0 / 6$ & $33.0(76.7)$ & $14.0(32.6)$ & $9.0(20.9)$ & $5.0(11.6)$ & $1.0(2.3)$ \\
\hline Cohen et al, $2015^{25}$ & 24 & $100 / 0 / 0 / 0$ & 19.0 (79.2) & $13.0(54.2)$ & $2.0(8.3)$ & $0.0(0.0)$ & $0.0(0.0)$ \\
\hline Lockau et al, $2015^{7}$ & 37 & $65 / 35 / 0 / 0$ & $27.0(73.0)$ & $17.0(45.9)$ & 7.0 (18.9) & $4.0(10.8)$ & $8.0(21.6)$ \\
\hline Spiotta et al, $2015^{26}$ & 16 & $100 / 0 / 0 / 0$ & $16.0(100.0)$ & $8.0(50.0)$ & $3.0(18.8)$ & $2.0(12.5)$ & $0.0(0.0)$ \\
\hline Lescher et al, $2015^{13}$ & 39 & $28 / 18 / 54 / 0$ & $25.0(64.1)$ & $14.0(35.9)$ & $4.0(10.3)$ & $4.0(10.3)$ & $0.0(0.0)$ \\
\hline Behme et al, $2015^{27}$ & 170 & $100 / 0 / 0 / 0$ & $130.0(76.5)$ & $62.0(36.5)$ & $32.0(18.8)$ & $15.0(8.8)$ & NA \\
\hline Grigoryan et al, $2016^{15}$ & 100 & $89 / 11 / 0 / 0$ & $88.0(88.0)$ & $42.0(42.0)$ & $20.0(20.0)$ & NA & NA \\
\hline Fahed et al, $2016^{14}$ & 70 & $54 / 19 / 19 / 8$ & $47.0(67.1)$ & $33.0(47.1)$ & $9.0(13.4)$ & NA & $12.0(17.1)$ \\
\hline This series & 86 & $77 / 23 / 0 / 0$ & $56.0(65.1)$ & $41.0(48.2)$ & $17.0(20.0)$ & $11.0(12.8)$ & $24.0(27.9)$ \\
\hline Atherothrombotic TOs & 66 & & $42.0(63.6)$ & $27.0(41.5)$ & $15.0(23.1)^{\prime}$ & 10.0 (15.2) & $22.0(33.3)$ \\
\hline Dissecting TOs ${ }^{19}$ & 20 & & $14.0(70.0)$ & $14.0(70.0)$ & $2.0(10.0)$ & $1.0(5.0)$ & $2.0(10.0)$ \\
\hline
\end{tabular}

Note:-NA indicates non-available data; TIMI, Thrombolysis in Myocardial Infarction.

a Distribution of the TO etiologies in percentages: atheromatous/dissection/cardioembolic/other causes.

${ }^{\mathrm{b}}$ Recanalization assessed with the TIMI score

3 months (mRS $0-2=45.9 \%$ in the HERMES analysis versus $41.5 \%$ in our cohort). However, our analysis differs from that in the HERMES meta-analysis as well as in previous studies reporting, specifically, experiences in the endovascular management of tandem occlusions in that we compared the atherothrombotic tandem occlusions with dissection-related tandem occlusions and isolated intracranial occlusions. This work provides new knowledge on TO pathology comprehension, which should help in patient management and future study designs. Indeed, the different pathomechanisms of extracranial ICA lesions involved in TOs could call for specific selection criteria and different recanalization strategies according to the TO etiology.

Our work has several limitations. It was a retrospective study with limited number of patients, though data were collected prospectively and consecutively. As a single-center study, our work has an inherent population bias. Therefore, our results cannot be generalized, though this is the largest reported series of atherothrombotic TOs selected by MR imaging, to our knowledge. Moreover, our work does not allow concluding on the safety of emergent carotid angioplasty and stent placement because of the small number of treated patients. However, intrastent thrombosis occurred more frequently in patients stented at the acute setting and was clinically symptomatic in $40 \%$ of them with poor clinical outcomes. The grading scale of the proximal ICA stenosis due to atherosclerotic plaque was arbitrary, defined to facilitate the analysis and make the results clearer. However, an underestimation of the grade 2 plaques and an overestimation of the grade 3 plaques cannot be excluded because of the potential iatrogenic ulceration that can be induced during the crossing of the proximal occlusion site.

\section{CONCLUSIONS}

Atherothrombotic TOs are a particularly complex and challenging cause of AIS. Compared with dissection-related TOs and isolated intracranial occlusions, they are associated with significantly lower successful reperfusion rates, lower favorable outcomes, and higher rates of procedural complications. Our results support the need to differentiate TOs according to their etiology when designing prospective studies on AIS management. The results of future trials and multicenter studies on specifically this stroke subpopulation are eagerly awaited.
Disclosures: Omer F. Eker-UNRELATED: Consultancy: Stryker; Payment for Educational Presentations: Covidien; Payment for Development of Educational Presentations: Covidien, Medtronic, Stryker. Vincent Costalat-UNRELATED: Grants/ Grants Pending: Medtronic; Consultancy: Sequent Medical, Balt, Codman, Stryker; Payment for Lectures (including Service on Speakers Bureaus): Stryker, Balt; Payment for Development of Educational Presentations: Covidien, Stryker, Medtronic. Alain Bonafé-UNRELATED: Consultancy: Medtronic, Stryker, Covidien, ev3; Grants/Grants Pending: Covidien*. *Money paid to the institution.

\section{REFERENCES}

1. Berkhemer OA, Fransen PS, Beumer D, et al. A randomized trial of intraarterial treatment for acute ischemic stroke. NEngl J Med 2015; 372:11-20 CrossRef Medline

2. Saver JL, Goyal M, Bonafe A, et al; SWIFT PRIME Investigators. Stent-retriever thrombectomy after intravenous t-PA vs. t-PA alone in stroke. $N$ Engl J Med 2015;372:2285-95 CrossRef Medline

3. Goyal M, Demchuk AM, Menon BK, et al; ESCAPE Trial Investigators. Randomized assessment of rapid endovascular treatment of ischemic stroke. N Engl J Med 2015;372:1019-30 CrossRef Medline

4. Campbell BC, Mitchell PJ, Kleinig TJ, et al; EXTEND-IA Investigators. Endovascular therapy for ischemic stroke with perfusion-imaging selection. $N$ Engl J Med 2015;372:1009-18 CrossRef Medline

5. Jovin TG, Chamorro A, Cobo E, et al; REVASCAT Trial Investigators. Thrombectomy within $\mathbf{8}$ hours after symptom onset in ischemic stroke. N Engl J Med 2015;372:2296-306 CrossRef Medline

6. Adams HP Jr, Bendixen BH, Kappelle LJ, et al. Classification of subtype of acute ischemic stroke: definitions for use in a multicenter clinical trial-TOAST. Trial of Org 10172 in Acute Stroke Treatment. Stroke 1993;24:35-41 CrossRef Medline

7. Lockau H, Liebig T, Henning T, et al. Mechanical thrombectomy in tandem occlusion: procedural considerations and clinical results. Neuroradiology 2015;57:589-98 CrossRef Medline

8. Benninger DH, Georgiadis D, Kremer C, et al. Mechanism of ischemic infarct in spontaneous carotid dissection. Stroke 2004;35: 482-85 CrossRef Medline

9. Kim YS, Garami Z, Mikulik R, et al. Early recanalization rates and clinical outcomes in patients with tandem internal carotid artery/ middle cerebral artery occlusion and isolated middle cerebral artery occlusion. Stroke 2005;36:869-71 CrossRef Medline

10. Rubiera M, Ribo M, Delgado-Mederos R, et al. Tandem internal carotid artery/middle cerebral artery occlusion: an independent predictor of poor outcome after systemic thrombolysis. Stroke 2006;37: 2301-05 CrossRef Medline

11. Blinc A, Francis CW. Transport processes in fibrinolysis and fibrinolytic therapy. Thromb Haemost 1996;76:481-91 Medline

12. Molina CA, Montaner J, Arenillas JF, et al. Differential pattern of tissue plasminogen activator-induced proximal middle cerebral ar- 
tery recanalization among stroke subtypes. Stroke 2004;35:486-90 CrossRef Medline

13. Lescher S, Czeppan K, Porto L, et al. Acute stroke and obstruction of the extracranial carotid artery combined with intracranial tandem occlusion: results of interventional revascularization. Cardiovasc Intervent Radiol 2015;38:304-13 CrossRef Medline

14. Fahed R, Redjem H, Blanc R, et al. Endovascular management of acute ischemic strokes with tandem occlusions. Cerebrovasc Dis 2016;41:298-305 CrossRef Medline

15. Grigoryan M, Haussen DC, Hassan AE, et al. Endovascular treatment of acute ischemic stroke due to tandem occlusions: large multicenter series and systematic review. Cerebrovasc Dis 2016;41: 306-12 CrossRef Medline

16. Goyal M, Menon BK, van Zwam WH, et al; HERMES collaborators. Endovascular thrombectomy after large-vessel ischaemic stroke: a meta-analysis of individual patient data from five randomised trials. Lancet 2016;387:1723-31 CrossRef Medline

17. Hacke W, Kaste M, Fieschi C, et al. Intravenous thrombolysis with recombinant tissue plasminogen activator for acute hemispheric stroke: the European Cooperative Acute Stroke Study (ECASS). JAMA 1995;274:1017-25 CrossRef Medline

18. Zaidat OO, Yoo AJ, Khatri P, et al; Cerebral Angiographic Revascularization Grading (CARG) Collaborators, STIR Revascularization working group, STIR Thrombolysis in Cerebral Infarction (TICI) Task Force. Recommendations on angiographic revascularization grading standards for acute ischemic stroke: a consensus statement. Stroke 2013;44:2650-63 CrossRef Medline

19. Marnat G, Mourand I, Eker O, et al. Endovascular management of tandem occlusion stroke related to internal carotid artery dissection using a distal to proximal approach: insight from the RECOST study. AJNR Am J Neuroradiol 2016;37:1281-88 CrossRef Medline

20. Team RC. R: A Language and Environment for Statistical Computing. Vienna: R Foundation for Statistical Computing; 2017

21. Malik AM, Vora NA, Lin R, et al. Endovascular treatment of tandem extracranial/intracranial anterior circulation occlusions: preliminary single-center experience. Stroke 2011;42:1653-57 CrossRef Medline

22. Kwak HS, Hwang SB, Jin GY, et al. Predictors of functional outcome after emergency carotid artery stenting and intra-arterial thrombolysis for treatment of acute stroke associated with obstruction of the proximal internal carotid artery and tandem downstream occlusion. AJNR Am J Neuroradiol 2013;34:841-46 CrossRef Medline

23. Stampfl S, Ringleb PA, Möhlenbruch M, et al. Emergency cervical internal carotid artery stenting in combination with intracranial thrombectomy in acute stroke. AJNR Am J Neuroradiol 2014;35: 741-46 CrossRef Medline

24. Maurer CJ, Joachimski F, Berlis A. Two in one: endovascular treatment of acute tandem occlusions in the anterior circulation. Clin Neuroradiol 2015;25:397-402 CrossRef Medline

25. Cohen JE, Gomori JM, Rajz G, et al. Extracranial carotid artery stenting followed by intracranial stent-based thrombectomy for acute tandem occlusive disease. J Neurointerv Surg 2015;7:412-17 CrossRef Medline

26. Spiotta AM, Lena J, Vargas J, et al. Proximal to distal approach in the treatment of tandem occlusions causing an acute stroke. J Neurointerv Surg 2015;7:164-69 CrossRef Medline

27. Behme D, Mpotsaris A, Zeyen P, et al. Emergency stenting of the extracranial internal carotid artery in combination with anterior circulation thrombectomy in acute ischemic stroke: a retrospective multicenter study. AJNR Am J Neuroradiol 2015;36:2340-45 CrossRef Medline

28. Sbarigia E, Toni D, Speziale F, et al. Emergency and early carotid endarterectomy in patients with acute ischemic stroke selected with a predefined protocol: a prospective pilot study. Int Angio 2003;22: 426-30 Medline

29. Abciximab Emergent Stroke Treatment Trial (AbESTT) Investigators. Emergency administration of abciximab for treatment of patients with acute ischemic stroke: results of a randomized phase 2 trial. Stroke 2005;36:880-90 CrossRef Medline

30. Heck DV, Brown MD. Carotid stenting and intracranial thrombectomy for treatment of acute stroke due to tandem occlusions with aggressive antiplatelet therapy may be associated with a high incidence of intracranial hemorrhage. J Neurointerv Surg 2015;7:170-75 CrossRef Medline 